\title{
Greater radial tuberosity size is associated with distal biceps tendon rupture: a quantitative 3-D CT case-control study
}

\author{
Nick F. J. Hilgersom ${ }^{1,2}\left(\right.$ D $\cdot$ Myrthe Nagel $^{1,2} \cdot$ Stein J. Janssen ${ }^{1} \cdot$ Izaäk F. Kodde $^{3} \cdot$ Bertram The $^{2} \cdot$ Denise Eygendaal $^{1,2}$
}

Received: 14 January 2021 / Accepted: 24 August 2021 / Published online: 4 September 2021

(c) The Author(s) 2021

\begin{abstract}
Purpose During pronation, the distal biceps tendon and radial tuberosity internally rotate into the radioulnar space, reducing the linear distance between the radius and ulna by approximately $50 \%$. This leaves a small space for the distal biceps tendon to move in and could possibly cause mechanical impingement or rubbing of the distal biceps tendon. Hypertrophy of the radial tuberosity potentially increases the risk of mechanical impingement of the distal biceps tendon. The purpose of our study was to determine if radial tuberosity size is associated with rupturing of the distal biceps tendon.

Methods Nine patients with a distal biceps tendon rupture who underwent CT were matched 1:2 to controls without distal biceps pathology. A quantitative 3-dimensional CT technique was used to calculate the following radial tuberosity characteristics: 1) volume in $\mathrm{mm}^{3}$, 2) surface area in $\mathrm{mm}^{2}, 3$ ) maximum height in $\mathrm{mm}$ and 4) location (distance in $\mathrm{mm}$ from the articular surface of the radial head).

Results Analysis of the 3-dimensional radial tuberosity CT-models showed larger radial tuberosity volume and maximum height in the distal biceps tendon rupture group compared to the control group. Mean radial tuberosity volume in the rupture-group was $705 \mathrm{~mm}^{3}$ (SD: $222 \mathrm{~mm}^{3}$ ) compared to $541 \mathrm{~mm}^{3}$ (SD: $184 \mathrm{~mm}^{3}$ ) in the control group $(p=0.033$ ). Mean radial tuberosity maximum height in the rupture-group was $4.6 \mathrm{~mm}$ (SD: $0.9 \mathrm{~mm}$ ) compared to $3.7 \mathrm{~mm}$ (SD: $1.1 \mathrm{~mm}$ ) in the control group, respectively $(p=0.011)$. There was no statistically significant difference in radial tuberosity surface area (ns) and radial tuberosity location (ns).

Conclusion Radial tuberosity volume and maximum height were significantly greater in patients with distal biceps tendon ruptures compared to matched controls without distal biceps tendon pathology. This supports the theory that hypertrophy of the radial tuberosity plays a role in developing distal biceps tendon pathology.
\end{abstract}

Level of evidence Level III.

Keywords Elbow $\cdot$ Biceps tendon $\cdot$ Rupture $\cdot$ Radial tuberosity $\cdot$ Size $\cdot$ Morphology $\cdot$ CT $\cdot$ 3D $\cdot$ Distal biceps tendon $\cdot$ Q3DCT · Impingement

\section{Introduction}

Nick F. J. Hilgersom

n.f.hilgersom@amsterdamumc.nl

1 Department of Orthopaedic Surgery, Amsterdam University Medical Centres, Location AMC, Meibergdreef 9, 1105 AZ Amsterdam, The Netherlands

2 Department of Orthopaedic Surgery, Amphia Hospital, 4819 EV Breda, The Netherlands

3 Department of Orthopaedic Surgery, St Antonius Hospital, 3543 AZ Utrecht, The Netherlands
Distal biceps tendon ruptures are more common than previously thought with an incidence of approximately 1.2-5.4 per 100,000 persons per year [8, 19]. Risk factors are male gender, smoking, use of steroids, and obesity [8, 19]. However, the exact pathophysiology of distal biceps tendon rupture remains unclear.

During pronation, the distal biceps tendon and radial tuberosity internally rotate towards the ulna, reducing the linear distance between the radius and ulna by approximately $45-48 \%[3,11,18,22]$ and leaving little space $(<1 \mathrm{~mm})$ for the biceps tendon to move [14]. This could possibly cause mechanical impingement of the distal biceps tendon. In 
1956, Davis and Yassine [4] were the first to suggest but as far as we know- never proved, that hypertrophic changes of the radial tuberosity decrease the radioulnar space, causing rubbing of the tendon, predisposing it to degenerative changes and finally rupture. Numerous studies have investigated the insertional footprint anatomy of the distal biceps tendon and radial tuberosity morphology to optimize surgical techniques for re-fixation of the distal biceps tendon and functional outcomes $[1,5-7,15,16,20]$. However, only Kodde et al. [10] have investigated the possible role of radial tuberosity size in distal biceps tendon rupture. They compared radial tuberosity size between patients with a distal biceps tendon rupture and matched controls without distal biceps tendon pathology using anterior-posterior views of conventional radiographs. Radial tuberosity size was expressed as a ratio based on the maximum diameter of the radius at the radial tuberosity divided by the diameter of the radius just distal of the radial tuberosity. They found no difference in radial tuberosity size. However, their study method leaves room for improvement because the ratio used does not represent absolute radial tuberosity size and the use of 2-dimensional imaging is limited by variation in positioning of the patients' arm, and thereby incomplete appreciation of the 3-dimensional morphology of the radial tuberosity. Quantitative 3D CT analysis is a more accurate method to assess the morphology, dimensions and volume of bone [9, 17, 24]. Quantitative 3D CT has been used for determining fracture morphology in a wide range of fractures (e.g., glenoid, elbow, and spine fractures) [12, 13, 23, 24], but is also useful for determining lesion size and pattern of osteochondritis dissecans of for example the capitellum [2].

The aim of this study was to assess whether radial tuberosity size plays a role in the pathophysiology of distal biceps tendon ruptures, using quantitative 3D CT analysis. Our null-hypothesis was that there would be no difference in radial tuberosity size (maximum height, surface area, and volume) between patients with distal biceps tendon ruptures and patients without distal biceps tendon ruptures.

\section{Materials and methods}

Adult patients ( $\geq 18$ years of age) with a distal biceps tendon rupture who had a CT scan of the injured elbow before operative re-fixation were included. It is routine practice at our institution to perform a CT scan of the elbow in patients with a delayed presentation of a distal biceps tendon rupture (> 4 weeks of complaints).

Each patient with a rupture (i.e., case) was matched to two control patients without a rupture (i.e., control). Subjects were matched by age within 10-year range and sex. The control group was randomly retrieved from a database of patients who had a CT scan performed of the elbow in the same hospital and based on diagnostic and procedure codes including: elbow trauma (excluding the proximal radius) and degenerative conditions (i.e., osteoarthritis). Indications for control CT scans were: degenerative disease $(n=5)$, trauma $(n=12)$, tumor $(n=1)$.

Patients (cases and controls) were excluded if they had a pre-existing elbow disease believed to affect proximal radius morphology (e.g., congenital disease, trauma).

\section{Outcome measures and demographics}

The medical records were reviewed to identify the baseline characteristics.

The outcome measures were: volume, surface area, maximum height, and location of the radial tuberosity. Therefore, 3D models were rendered using OsiriX medical image viewer application (version 10.0.4, Bernex, Switzerland). Cortical bone was automatically identified using a predefined Hounsfield unit value threshold of $>300$. The resolution was set highest, decimate-resolution at 0.1 , and smooth-iterations at 20 for every case. Subsequently these 3D polygon mesh models were imported in Rhinoceros (McNeel 5.0, Seattle, Washington) for measurements. The position of the 3D models was standardized using the $x$-, $y$-, and $z$-axes in Rhinoceros (Fig. 1).

The shaft of the proximal radius was used to define proximal and distal ( $y$-axis), the radial tuberosity was used to define lateral and medial side ( $x$-axis), and anterior and posterior ( $z$-axis). A plane was created to separate the radial tuberosity from the proximal radius: this was done visually based on the up- and downslope at the beginning and end of the radial tuberosity in the sagittal plane,

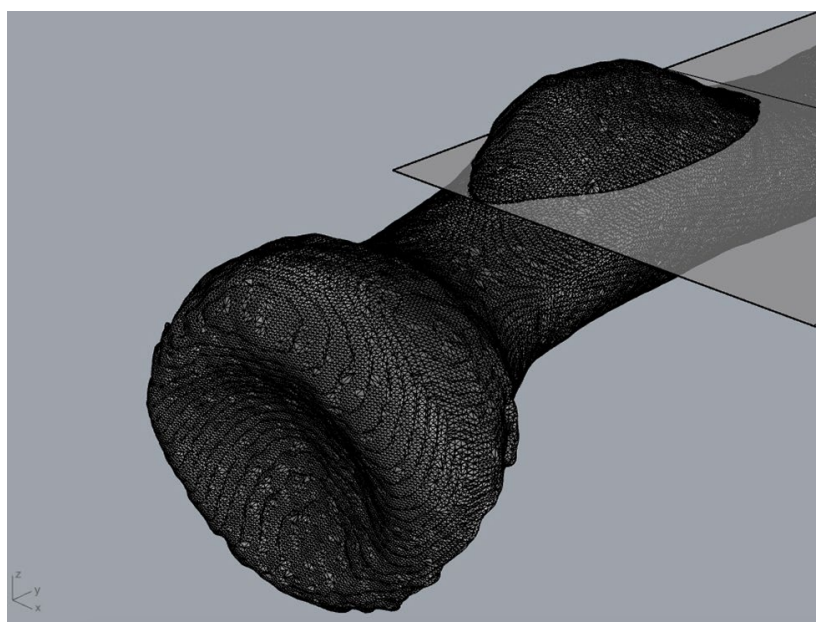

Fig. 1 Rhinoceros 3D model of the proximal radius demonstrating the plane created to separate the radial tuberosity from the proximal radial shaft to perform measurements on the radial tuberosity. The $y$-axis determines proximal-distal. The $x$-axis and $z$-axis determine lateral-medial and anterior-posterior, respectively 
subsequently the proximal radius was rotated in the axial plane to ascertain that the radial tuberosity lied above the plane (Fig. 1). The researcher (SJJ) who performed the measurements and created the heatmaps was not involved in patient care and blinded for group assignment (case versus control) to avoid bias in measurements. Group assignment was revealed at time of final statistical analysis.

The volume of the radial tuberosity was measured in $\mathrm{mm}^{3}\left(\mathrm{~mm}=\right.$ millimeter), the surface area in $\mathrm{mm}^{2}$ (Fig. 2A), the maximum height in $\mathrm{mm}$ (Fig. 2B) and the location as a distance in $\mathrm{mm}$ measured from the volumetric center of the radial tuberosity to the center of the radial head articular surface (Fig. 2C). A point grid with $x, y$, and $z$ coordinates of the radial tuberosity was extracted for further analyses of the radial tuberosity profile. These values were imported per radial tuberosity in Stata 15.0 (StataCorp LP, College Station, TX). Subsequently an averaged radial tuberosity profile heatmap was created for both the cases (distal biceps tendon ruptures) versus controls (no distal biceps tendon rupture) and for each radial tuberosity separately.

Our institutional review board approved this retrospective imaging study (Medical Ethics Committee of the Amphia Hospital; N2018-0131). Informed consent was not required for this retrospective study.

\section{Statistical analysis}

Categorical variables are reported as frequencies and percentages, and continuous variables as mean with standard deviation (SD). To account for paired data, we used mixed-effects linear regression with random effects for the case-control matched groups and fixed effects for the group assignment to calculate $p$ values and assess if there was a significant difference in the continuous outcome measures between the cases and the controls. The results of mixed-effects linear regression are interpreted like those derived from a linear regression model.
A two-tailed $p$ value below 0.05 was considered statistically significant and Stata was used for all statistical analysis. There were no missing values for any of the variables.

A post hoc power analysis demonstrated that with the included 9:18 matched cases and controls, given the means and standard deviations, and a correlation of 0.1 , sufficient power $(\beta=0.85)$ was achieved to detect a significant difference in volume of the radial tuberosity. In addition, sufficient power $(\beta=0.97)$ was achieved to detect a significant difference in max radial tuberosity height. Insufficient power $(\beta<0.80)$ was achieved to detect a significant difference in area $(\beta=0.79)$ and location $(\beta=0.77)$ of the radial tuberosity.

\section{Results}

Nine consecutive patients who routinely had a CT scan (0.75-1.00 mm slice thickness, $80 \mathrm{kV}, 55-57 \mathrm{mAs})$ of their elbow performed after a subacute or chronic distal biceps tendon rupture and before operative re-fixation were identified at our institution between October 2015 and March 2018. Five patients had a partial distal biceps tendon rupture, and four patients had a complete distal biceps tendon rupture. No patients had to be excluded based on pre-existing elbow disease believed to affect proximal radius morphology. The cohort consisted of nine cases and 18 sex- and agematched controls with adequate $\mathrm{CT}$ scans for 3D modeling.

The overall mean age was 47 (SD: 10) years for the cases and 46 (SD: 10) years for the controls (Table 1). The age difference was found to be statistically significant $(p=0.014)$. The majority was male: $89 \%$ (24/27). There was no statistically significant difference in height, weight or BMI between cases and controls.

Analysis of the 3D radial tuberosity CT-models showed larger radial tuberosity volume and maximum height in the distal biceps tendon rupture group as compared to the control group (Table 2). Mean radial tuberosity volume in the rupture-group was $705 \mathrm{~mm}^{3}$ (SD: $222 \mathrm{~mm}^{3}$ ) compared to

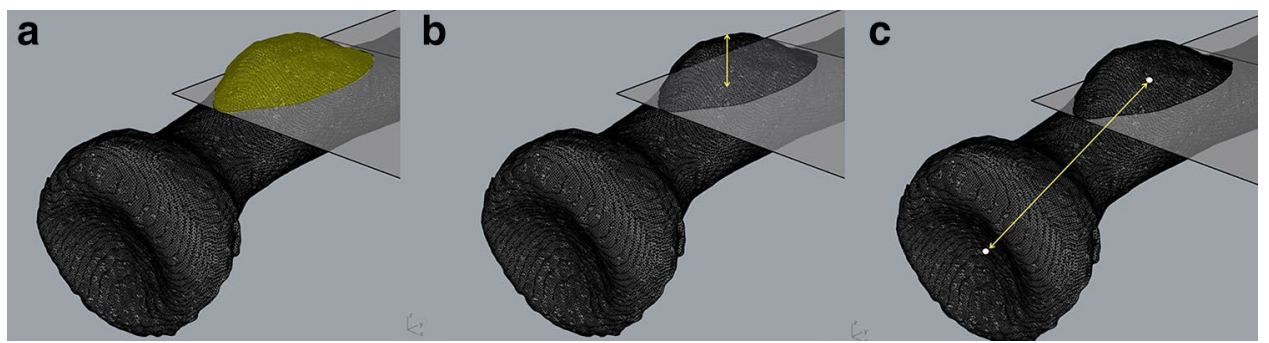

Fig. 2 Rhinoceros 3D model of the proximal radius demonstrating the measurements performed on the radial tuberosity. A Volume and surface area (marked in yellow), $\mathbf{B}$ maximum height (greatest distance between the plane separating the radial tuberosity from the proximal radius and top of the radial tuberosity), and $\mathbf{C}$ location (the distance in $\mathrm{mm}$ measured from the volumetric center of the radial tuberosity to the center of the radial head articular surface) 
Table 1 Baseline characteristics of cases (ruptures) and controls (no ruptures)

\begin{tabular}{|c|c|c|c|c|}
\hline & $\begin{array}{l}\text { Overall }(n=27) \\
\text { Mean }( \pm S D)\end{array}$ & $\begin{array}{l}\text { Rupture }(n=9) \\
\text { Mean }( \pm \text { SD })\end{array}$ & $\begin{array}{l}\text { Controls }(n=18) \\
\text { Mean }( \pm \text { SD })\end{array}$ & $p$ value \\
\hline Age (in years) & $47(10)$ & $47(10)$ & $46(10)$ & 0.014 \\
\hline Height (in $\mathrm{cm}$ )* & $179(9.4)$ & $179(9.1)$ & $179(10)$ & ns \\
\hline Weight (in kg)* & $82(16)$ & $86(17)$ & $79(16)$ & ns \\
\hline \multirow[t]{2}{*}{ BMI* } & $25(3.6)$ & $26(4.1)$ & $24(3.2)$ & ns \\
\hline & $n(\%)$ & $n(\%)$ & $n(\%)$ & $p$ value \\
\hline Male & $24(89)$ & $8(89)$ & $16(89)$ & ns \\
\hline Right arm affected & $16(59)$ & $5(56)$ & $11(61)$ & ns \\
\hline
\end{tabular}

$S D$ Standard deviation, $n s$ non-significant

*There were 5 missing values for length, weight, and BMI

Table 2 Difference in volume, surface area, maximum height and location of radial tuberosity measures between biceps ruptures and controls (no rupture)

\begin{tabular}{llll}
\hline & $\begin{array}{l}\text { Rupture }(\mathrm{n}=9) \\
\text { Mean }( \pm \mathrm{SD})\end{array}$ & $\begin{array}{l}\text { Controls }(\mathrm{n}=18) \\
\text { Mean }( \pm \mathrm{SD})\end{array}$ & $p$ value \\
\hline Volume $\left(\right.$ in $\left.\mathrm{mm}^{3}\right)$ & $705(222)$ & $541(184)$ & $\mathbf{0 . 0 3 3}$ \\
Area $\left(\right.$ in $\left.\mathrm{mm}^{2}\right)$ & $417(63)$ & $365(81)$ & $\mathrm{ns}$ \\
Max Height $($ in $\mathrm{mm})$ & $4.6(0.9)$ & $3.7(1.1)$ & $\mathbf{0 . 0 1 1}$ \\
Location (in mm) & $34(3.3)$ & $34(2.4)$ & $\mathrm{ns}$ \\
\hline
\end{tabular}

$S D$ Standard deviation, $n s$ non-significant
$541 \mathrm{~mm}^{3}$ (SD: $\left.184 \mathrm{~mm}^{3}\right)$ in the control group $(p=0.033)$. Mean radial tuberosity maximum height in the rupture group was $4.6 \mathrm{~mm}$ (SD: $0.9 \mathrm{~mm}$ ) compared to $3.7 \mathrm{~mm}$ (SD: $1.1 \mathrm{~mm})$ in the control group, respectively $(p=0.011)$ (Fig. 3). There was no statistically significant difference in radial tuberosity surface area (n.s.) and radial tuberosity location (n.s.) with the current sample size.

\section{Discussion}

The presented data, obtained using a quantitative 3D CT technique, show that radial tuberosity volume and maximum height are greater in patients with distal biceps tendon ruptures compared to patients without distal biceps tendon

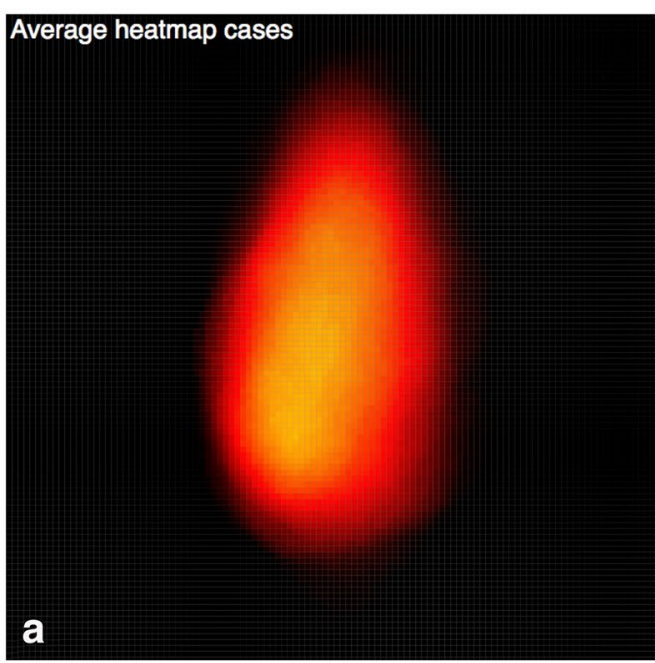

Fig. 3 Heatmaps demonstrating the averaged radial tuberosity height profile of cases who had a distal biceps tendon rupture (A) versus controls who did not have a distal biceps tendon rupture (B). The length of the $x$-axis and $y$-axis are $36.7 \mathrm{~mm}$. The top of the graph is

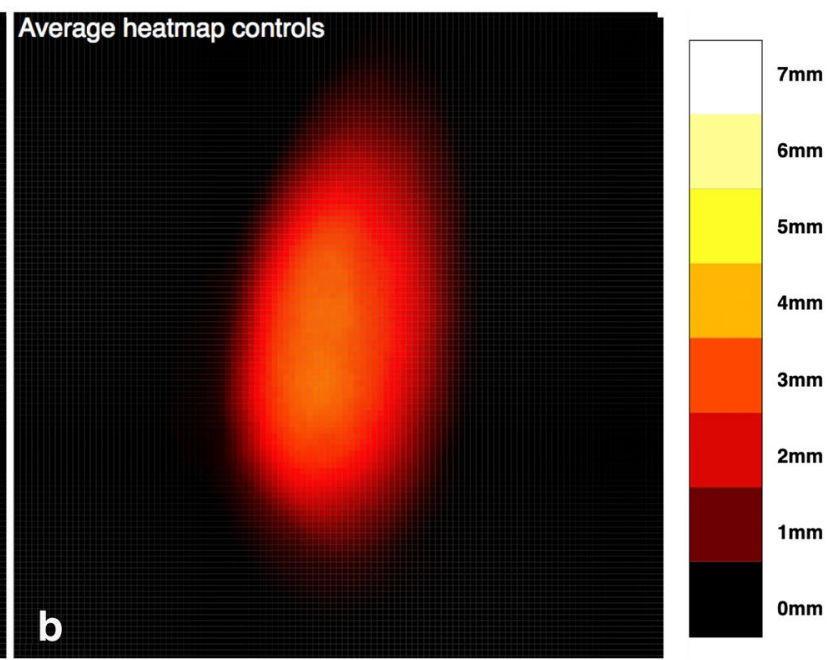

distal, the bottom proximal. All images are right-sided radial tuberosities (left-sided radial tuberosities are mirrored). The color intensity scale indicates the height of the radial tuberosity, $0 \mathrm{~mm}$ (black) to $7 \mathrm{~mm}$ (white) 
pathology. No significant differences were found for radial tuberosity surface area and location with the current sample size. The greater radial tuberosity volume and maximum height in patients with distal biceps tendon ruptures support the theory that hypertrophic changes of the radial tuberosity may play a role in distal biceps tendon pathology. Therefore, the null-hypothesis that there would be no difference in radial tuberosity volume and maximum height was rejected.

Current results are in contrast to the findings in the study by Kodde et al. [10], who found no significant difference in radial tuberosity size between patients with a distal biceps tendon rupture and matched controls without distal biceps tendon pathology. The difference in results may be explained by two factors: use of a ratio to represent radial tuberosity size, and the used imaging technique. First, Kodde et al. [10] used a ratio to reflect radial tuberosity size that was determined by the maximum diameter of the radius at the radial tuberosity to the diameter of the diaphysis just distal to the radial tuberosity. A drawback of using a ratio is that it is a derivative and not an exact representation of size, therefore less accurate. Second, measurements were performed on conventional AP-views of the elbow, which only provides information in a 2-dimensional plane. Although the method of obtaining the AP-views was standardized, the measured radial tuberosity height would be depending on the angle at which the radial tuberosity was depicted, which can be influenced by patient's forearm position, anatomic variability in radial tuberosity location between patients and angle of the $\mathrm{X}$-ray beam. Maybe with use of fluoroscopy, it would have been possible to consistently get the plane at which the radial tuberosity is cut at its maximum height; any other angle would show a smaller tuberosity height and lead to underestimation of radial tuberosity size. In this study, both concerns were addressed using 3D CT analysis which provided an accurate 3D-model of the radial tuberosity allowing exact measurements of radial tuberosity size, including height, surface and volume, as well as morphology assessment.

Based on previously published literature, we believe the role of the radial tuberosity in distal biceps tendon pathophysiology can be twofold; causative or reactive. A larger and higher radial tuberosity may directly cause rubbing of the distal biceps tendon during pronation [4], or narrow the radioulnar space causing the distal biceps tendon to become wedged in between the radial tuberosity and lateral ulna [3, $14,22]$. The latter has also been described as a risk factor for re-rupture after distal biceps tendon reconstruction [11]. Both ways of mechanical impingement can cause recurring micro-trauma to the distal biceps tendon, predisposing it to degenerative changes and finally rupture. Reversely, one can also reason from Wolff's law [25] that the hypertrophic changes of the radial tuberosity are caused by overload of the biceps muscle causing micro-trauma to the distal biceps tendon and radial tuberosity due to traction forces. This may eventually lead to a vicious circle in which micro-trauma causes the radial tuberosity to increase in size, and the hypertrophic radial tuberosity in turn attenuates the distal biceps tendon.

The involvement of radial tuberosity volume and maximum height in distal biceps tendon pathophysiology may have consequences for distal biceps tendon repair technique: i.e., site of tendon reattachment, choice of re-fixation technique and whether or not to reduce radial tuberosity height. One could reason that radial tuberosity height should be reduced during surgery, as such increasing radioulnar space, to prevent mechanical impingement of the reconstructed biceps tendon. However, Schmidt et al. [21] have shown in a cadaveric study that a $25 \%$ loss of radial tuberosity height resulted in a significant $27 \%$ lower supination moment arm at $60^{\circ}$ supination. The absolute difference in maximum height between the rupture group and control group is $0.9 \mathrm{~mm}(4.6-3.7 \mathrm{~mm})$, this is approximately $20-24 \%$ of the radial tuberosity height. Whether this would result in a clinically relevant loss of supination force was not investigated. Therefore, simply reducing the height of the radial tuberosity in all cases is not an option and further investigation is needed.

This study has some limitations. First, this study is limited by a relatively small number of patients which could induce selection bias and limit power. However, CT scanning is performed routinely in patients with chronic distal biceps ruptures at our institution and patients were included in a consecutive fashion reducing the chance of selection bias. Next, quantitative 3D CT analysis is highly accurate limiting the required sample size. To maximize statistical power, cases were matched to controls on a 1:2 basis. A post hoc power analysis demonstrated sufficient power to detect a significant difference in volume and height of the radial tuberosity, and insufficient power to detect a significant difference in area and location of the radial tuberosity. A slightly larger sample size might have resulted in significant differences in radial tuberosity area and location. A significant but small, clinically non-relevant, age difference of 1 year was found between the controls and cases. This was possible as subjects were matched by age within a 10-year range. Second, this is a retrospective study that does not consider all possible confounders. To limit this, matching was performed on the most common available confounders; age and gender. Unfortunately, it was not possible to match for arm dominance, smoking or BMI (height and weight) as these variables were not available for controls in the CT database prior to matching. These factors should be included in future (prospective) studies.

The strength of the current study is the use of a quantitative CT measurement technique that allowed accurate 3D assessment of radial tuberosity characteristics (volume, surface area and position) $[9,17,24]$. 
This is the first study that shows that radial tuberosity size is associated with the occurrence of distal biceps tendon ruptures, which may have implications for surgical reconstruction (whether or not to reduce radial tuberosity height) and understanding of distal biceps tendon rupture pathophysiology. Whether the role of the radial tuberosity is causative (i.e., mechanical impingement), reactive (i.e., hypertrophy due to recurring micro-traumata) or a combination of both remains to be clarified and warrants further investigation.

It is recommended to assess the radial tuberosity during the surgical procedure, prior to reinsertion, for hypertrophy. Remove any hypertrophic changes, spurs or cortical irregularities if present, but not simply to reduce radial tuberosity height.

\section{Conclusion}

Radial tuberosity volume and maximum height were significantly greater in patients with chronic distal biceps tendon ruptures compared to matched controls without distal biceps tendon pathology. This supports the theory that hypertrophy of the radial tuberosity plays a role in developing distal biceps tendon pathology.

Author contributions NFJH was responsible for study design, data interpretation, manuscript preparation, manuscript design, creation of figures and editing. $\mathrm{MN}$ was responsible for data collection, data analysis, manuscript preparation and editing. SJJ was responsible for study design, data collection, data analysis, data interpretation, creation of figures, manuscript preparation and editing. IFK and BT were responsible for data interpretation, clinical insight and providing feedback on the manuscript. DE were responsible for study design, data interpretation, clinical insight and providing feedback on the manuscript. All authors read and approved the final manuscript.

Funding No funding was received for this study.

\section{Declarations}

Conflict of interest DE receives grants for institutional research support from Zimmer-Biomet, Stryker and Matthys, personal fees for educational activities from A.O. and grants for OCD research support from ISAKOS, all outside of the submitted work. The remaining authors have no conflict of interest in the authorship or publication of this contribution.

Ethics approval This study was approved by the Medical Ethics Committee of the Amphia Hospital; N2018-0131.

Informed consent Not applicable.

Open Access This article is licensed under a Creative Commons Attribution 4.0 International License, which permits use, sharing, adaptation, distribution and reproduction in any medium or format, as long as you give appropriate credit to the original author(s) and the source, provide a link to the Creative Commons licence, and indicate if changes were made. The images or other third party material in this article are included in the article's Creative Commons licence, unless indicated otherwise in a credit line to the material. If material is not included in the article's Creative Commons licence and your intended use is not permitted by statutory regulation or exceeds the permitted use, you will need to obtain permission directly from the copyright holder. To view a copy of this licence, visit http://creativecommons.org/licenses/by/4.0/.

\section{References}

1. Athwal GS, Steinmann SP, Rispoli DM (2007) The distal biceps tendon: footprint and relevant clinical anatomy. J Hand Surg Am 32:1225-1229

2. Bexkens R, Oosterhoff JH, Tsai TY, Doornberg JN, van den Bekerom MPJ, Eygendaal D, Oh LS (2017) Osteochondritis dissecans of the capitellum: lesion size and pattern analysis using quantitative 3-dimensional computed tomography and mapping technique. J Shoulder Elbow Surg 26:1629-1635

3. Bhatia DN, Kandhari V, DasGupta B (2017) Cadaveric study of insertional anatomy of distal biceps tendon and its relationship to the dynamic proximal radioulnar space. J Hand Surg Am 42:e15-e23

4. Davis WM, Yassine Z (1956) An etiological factor in tear of the distal tendon of the biceps brachii; report of two cases. J Bone Jt Surg Am 38-A:1365-1368

5. Eames MH, Bain GI, Fogg QA, van Riet RP (2007) Distal biceps tendon anatomy: a cadaveric study. J Bone Jt Surg Am 89:1044-1049

6. Forthman CL, Zimmerman RM, Sullivan MJ, Gabel GT (2008) Cross-sectional anatomy of the bicipital tuberosity and biceps brachii tendon insertion: relevance to anatomic tendon repair. J Shoulder Elbow Surg 17:522-526

7. Hutchinson HL, Gloystein D, Gillespie M (2008) Distal biceps tendon insertion: an anatomic study. J Shoulder Elbow Surg 17:342-346

8. Kelly MP, Perkinson SG, Ablove RH, Tueting JL (2015) Distal biceps tendon ruptures: an epidemiological analysis using a large population database. Am J Sports Med 43:2012-2017

9. Kim G, Jung HJ, Lee HJ, Lee JS, Koo S, Chang SH (2012) Accuracy and reliability of length measurements on threedimensional computed tomography using open-source OsiriX software. J Digit Imaging 25:486-491

10. Kodde IF, van den Bekerom MP, Mulder PG, Eygendaal D (2016) The size of the radial tuberosity is not related to the occurrence of distal biceps tendon ruptures: a case-control study. Open Orthop J 10:1-6

11. Krueger CA, Aden JK, Broughton K, Rispoli DM (2014) Radioulnar space available at the level of the biceps tuberosity for repaired biceps tendon: a comparison of 4 techniques. J Shoulder Elbow Surg 23:1717-1723

12. Lee HD, Jung YJ, Oh JK, Moon JG (2021) Morphological characteristics of fractures of the anteromedial facet of the coronoid in posteromedial rotatory instability of the elbow: a three-dimensional CT remodeling study. J Shoulder Elbow Surg 30:1527-1536

13. Lubberts B, Janssen S, Mellema J, Ring D (2016) Quantitative 3-dimensional computed tomography analysis of olecranon fractures. J Shoulder Elbow Surg 25:831-836

14. Matsuki K, Sugaya H, Takahashi N, Tokai M, Ueda Y, Hoshika S, Hamada H, Banks SA (2020) Three-dimensional measurement of proximal radioulnar space during active forearm pronation. J Biomech 113:110120

15. Mazzocca AD, Burton KJ, Romeo AA, Santangelo S, Adams DA, Arciero RA (2007) Biomechanical evaluation of 4 techniques of distal biceps brachii tendon repair. Am J Sports Med $35: 252-258$ 
16. Mazzocca AD, Cohen M, Berkson E, Nicholson G, Carofino BC, Arciero R, Romeo AA (2007) The anatomy of the bicipital tuberosity and distal biceps tendon. J Shoulder Elbow Surg $16: 122-127$

17. Meijer DT, de Muinck Keizer RO, Stufkens SAS, Schepers T, Sierevelt IN, Kerkhoffs G, Goslings JC, Doornberg JN (2019) Quantification of postoperative posterior malleolar fragment reduction using 3-dimensional computed tomography (Q3DCT) determines outcome in a prospective pilot study of patients with rotational type ankle fractures. J Orthop Trauma 33:404-410

18. Rausch V, Krieter JP, Leschinger T, Hackl M, Scaal M, Muller LP, Wegmann K (2020) The radioulnar distance at the level of the radial tuberosity. Clin Anat 33:661-666

19. Safran MR, Graham SM (2002) Distal biceps tendon ruptures: incidence, demographics, and the effect of smoking. Clin Orthop Relat Res 404:275-283

20. Schmidt CC, Brown BT, Qvick LM, Stacowicz RZ, Latona CR, Miller MC (2016) Factors that determine supination strength following distal biceps repair. J Bone Jt Surg Am 98:1153-1160

21. Schmidt CC, Brown BT, Williams BG, Rubright JH, Schmidt DL, Pic AC, Nakashian MR, Schimoler PJ, Miller MC (2015) The importance of preserving the radial tuberosity during distal biceps repair. J Bone Jt Surg Am 97:2014-2023
22. Seiler JG 3rd, Parker LM, Chamberland PD, Sherbourne GM, Carpenter WA (1995) The distal biceps tendon. Two potential mechanisms involved in its rupture: arterial supply and mechanical impingement. J Shoulder Elbow Surg 4:149-156

23. ter Meulen DP, Janssen SJ, Hageman MG, Ring DC (2016) Quantitative three-dimensional computed tomography analysis of glenoid fracture patterns according to the AO/OTA classification. J Shoulder Elbow Surg 25:269-275

24. Verbeek BM, Janssen SJ, Pielkenrood BJ, Schwab JH (2020) Quantitative 3-dimensional Computed Tomography (Q3DCT) analysis of odontoid fractures. J Clin Neurosci 71:164-169

25. Wolff $\mathbf{J}$ (1988) Concerning the interrelationship between form and function of the individual parts of the organism. By Julius Wolff, 1900. Clin Orthop Relat Res 228:2-11

Publisher's Note Springer Nature remains neutral with regard to jurisdictional claims in published maps and institutional affiliations. 